\title{
A adiposidade corporal e a idade prejudicam a capacidade funcional para realizar as atividades da vida diária de mulheres acima de 47 anos
}

\author{
Vagner Raso \\ Departamento de Fisiopatologia Experimental - Faculdade de Medicina - USP
}

\section{RESUMO}

Este estudo teve como objetivo verificar o efeito da idade, peso, altura, índice de massa corporal e adiposidade corporal na capacidade funcional de mulheres acima de 47 anos para realizar as atividades da vida diária. Para tanto, a amostra foi constituída por 129 mulheres na faixa etária de 47 a 77 anos de idade. A adiposidade total (Adp T) foi considerada por meio do somatório das dobras cutâneas bíceps (BI), tríceps (TRI), subescapular (SB), axilar média (AM), supra-ilíaca (SI), abdominal (ABD), coxa anterior (CA) e panturrilha medial (PM). A adiposidade central (Adp C) representou o somatório de $\mathrm{SB}, \mathrm{AM}$, SI e $\mathrm{ABD}$, e a adiposidade periférica (Adp P), o somatório de BI, TRI, CA e PM. A capacidade funcional para realizar as atividades da vida diária foi predita mediante os testes: velocidade para se levantar de uma posição sentada (VLPS) e para se levantar de uma posição deitada (VLPD), velocidade para calçar e amarrar o tênis (VCAT) e velocidade para subir escada (VSE). Foi calculado o quartil $(Q)$ para todas as variáveis independentes (idade, peso, altura, índice de massa corporal e adiposidade corporal [total, central e periférica]). A análise estatística utilizada foi a ANOVA one way e os coeficientes de correlação linear de Pearson e Spearman rank-order. As diferenças estatisticamente significativas ocorreram principalmente entre os quartis das extremidades $\left(Q_{1} \times Q_{4}\right)$ para VLPS e VLPD (exceção para peso e IMC), enquanto para VSE foi entre $Q_{2}$ x $Q_{4}$ (idade). As variáveis independentes exerceram efeito nulo sobre VCAT. A magnitude de varia-

Recebido em 30/3/02

2a versão recebida em 22/6/02

Aceito em 8/8/02

Endereço para correspondência:

Rua Marquês de Praia Grande, 540, apto. 32B

03129-110 - São Paulo, SP

E-mail: vraso@usp.br ção percentual ocorreu entre a amplitude de $14,1 \%$ (idade [VLPD: $Q_{3} \times Q_{4}$ ]) e $36,1 \%$ (Adp T [VLPS: $Q_{2} \times Q_{4}$ ]). Em ordem, as variáveis independentes que exerceram maior repercussão negativa sobre a performance nos testes de capacidade funcional (VLPS, VLPD, VSE) para realizar as atividades da vida diária foram Adp C, idade, Adp T, Adp $\mathrm{P}$, IMC e peso.

Palavras-chave: Adiposidade corporal. Capacidade funcional. Composição corporal.

\section{ABSTRACT \\ Body adiposity and age impair the capacity to perform daily living activities of women older than 47 years}

The purpose of this study was to verify the effect of chronological age, body weight, body height, body mass index, and body adiposity on the functional capacity to perform daily living activities of women with $>47$ years of age. The sample comprised 129 women from 47 to 77 years of age. The total body adiposity (TBA) was represented by the sum total of biceps (BI), triceps (TRI), subscapular (SB), middle axillary (MA), suprailiac (SI), abdominal (ABD), anterior thigh (AT), and middle calf $(M C)$ skinfolds. Central body adiposity (CBA) was taken as the sum total of $S B$, $M A, S I$, and $A B D$, whereas peripheral body adiposity (PBA) was represented by the sum total of BI, TRI, AT and MC. The performance of daily living activities was estimated by: velocity to move from sitting to standing position (VSSP ${ }_{1}$ ), velocity to move from supine to standing position (VSSP ${ }_{2}$ ), velocity to climb stairs (VCS), and velocity to put on snickers and tie the laces (VWS). The quartile (Q) was calculated for all independent variables (chronological age, body weight, body height, body mass index, and body adiposity [total, central and peripheral]). The statistical analysis used was ANOVA "One Way" with post-hoc Bonferroni, and the coefficient of linear correlation (Pearson and Spearman Rank-Order). Statistical differences occurred specially 
between quartiles of extremities $\left(\mathrm{Q}_{1} \times \mathrm{Q}_{4}\right)$ for $\operatorname{VSSP}_{1}$ and $\mathrm{VSSP}_{2}$ (except for body weight and BMI), while for VCS it was between $\mathrm{Q}_{2} x \mathrm{Q}_{4}$ (chronological age). The independent variables exerted no effect on VWS. The percent range was from $14.1 \%$ (chronological age $\left[\operatorname{VSSP}_{2}: \mathrm{Q}_{3} \times \mathrm{Q}_{4}\right]$ ) to $36.1 \%\left(T B A\left[\operatorname{VSSP}_{1}: \mathrm{Q}_{2} x \mathrm{Q}_{4}\right]\right)$. Variables that exerted negative repercussion on functional capacity to perform daily living activities were central body adiposity, chronological age, total body adiposity, peripheral body adiposity, $B M I$ and body weight, in that order.

Key words: Body composition. Body adiposity. Functional capacity.

\section{INTRODUÇÃO}

A capacidade funcional para realizar as atividades mais comezinhas e/ou mais específicas da vida diária tem sido alvo de preocupação internacional ${ }^{1,2} \mathrm{e}$, recentemente, tem evocado interesse nacional crescente ${ }^{3-7}$. Essa preocupação fundamenta-se devido ao fato de que a incapacidade funcional para realizar as atividades da vida diária é preditora de fragilidade ${ }^{8}$, de sinais clínicos precoces de demência ${ }^{9}$ assim como de mortalidade ${ }^{10}$.

O envelhecimento per se é associado ao decréscimo da força e da massa musculares (sarcopenia), que têm sido referidos como uma das principais causas de incapacidade funcional ${ }^{11}$. Todavia, o processo natural de envelhecimento também é caracterizado pelo incremento do conteúdo de gordura corporal ${ }^{12}$.

Nesse caso, está muito bem documentada a associação direta entre adiposidade corporal e prevalência de morbidade por distúrbios plurimetabólicos ${ }^{13}$, doenças cardiovasculares ${ }^{14}$, além de outras ${ }^{15}$. Também está clara a relação entre avanço da idade e incremento tanto da prevalência como da incidência de doenças crônico-degenerativas ${ }^{16} \mathrm{e}$ infecto-contagiosas, além de neoplasias ${ }^{17}$. Contudo, é relevante ressaltar que esse dualismo não representa o processo natural de envelhecimento.

Por outro lado, muito embora esteja bem estabelecido que a adiposidade corporal, especialmente a deposição centrípeta de gordura, tenha relação direta com a prevalência de inúmeras doenças, e tenha sido reconhecida a característica multifatorial da incapacidade funcional ${ }^{18}$, na fase atual do conhecimento, ainda não está completamente clara a influência da composição corporal sobre a capacidade funcional de pessoas para realizar as atividades da vida diária. Portanto, este estudo teve como objetivo verificar o efeito da idade, peso, altura, índice de massa corporal e adiposidade corporal (total, central e periférica) na capacidade funcional de mulheres acima de 47 anos para realizar as atividades da vida diária.

\section{CASUÍSTICA E MÉTODOS}

\section{Voluntárias}

A amostra foi constituída por 129 mulheres na faixa etária de 47 a 77 anos de idade que se submeteram voluntariamente a uma bateria de testes de aptidão física e de capacidade funcional realizada no período entre 1998 e 2001, durante o Estudo do Envelhecimento de Americana. As voluntárias foram recrutadas por meio de divulgação nos órgãos de comunicação local (rádio, jornal, televisão). Foram avaliadas cerca de 300 pessoas de ambos os sexos durante esse período; no entanto, somente aquelas que foram submetidas à medida das oito dobras cutâneas foram consideradas. Os homens não foram incluídos na análise por representarem um número limitado de voluntários.

As voluntárias foram informadas sobre os objetivos deste estudo assim como sobre os benefícios e possíveis riscos à saúde. Também foram informadas de que a participação no estudo era voluntária e que poderiam desistir a qualquer momento. Após estas orientações, um termo de consentimento foi obtido de cada voluntária.

\section{Adiposidade corporal}

A adiposidade corporal foi determinada por meio das dobras cutâneas de acordo com a padronização descrita por França e Vívolo ${ }^{19}$. A exceção foi a dobra cutânea coxa anterior, que seguiu a descrição feita por Harrisson et al. ${ }^{20}$. Foram utilizadas as dobras cutâneas bíceps, tríceps, subescapular, axilar média, suprailíaca, abdominal, coxa anterior e panturrilha medial. A adiposidade corporal total (Adp T) foi representada pelo somatório das oito dobras cutâneas supracitadas. A adiposidade corporal central (Adp C) foi estabelecida por meio do somatório das dobras cutâneas subescapular, axilar média, suprailíaca e abdominal, e a adiposidade corporal periférica (Adp P), pelo somatório das dobras cutâneas bíceps, tríceps, coxa anterior e panturrilha medial.

Os valores intra-avaliadores referentes à adiposidade corporal foram reproduzidos em 24 voluntárias $(18,6 \%)$ com intervalo de tempo de 24 a 48 horas entre as duas medidas. Todos os valores foram muito altos e estatisticamente significativos ( $\mathrm{p}<0,0001)$; para a adiposidade corporal total o valor foi de $0,98\left(r^{2}=0,96\right)$, enquanto para a adiposidade corporal central e periférica, os valores foram de $0,96\left(\mathrm{r}^{2}=\right.$ $0,92)$ e $0,98\left(r^{2}=0,96\right)$, respectivamente.

\section{Capacidade funcional}

Para medir a performance dos sujeitos nas atividades da vida diária foram selecionados e sugeridos os seguintes testes:

Velocidade para levantar-se de uma posição sentada (VLPS). O indivíduo senta-se em um colchonete de ginásti- 
ca com as pernas estendidas e encostadas uma à outra, as mãos sobre os joelhos. $\mathrm{O}$ avaliador fica ao lado direito do avaliado e através da voz de comando "Atenção! Já!" inicia o teste, acionando concomitantemente o cronômetro. $\mathrm{O}$ avaliado deve levantar-se da maneira que lhe for mais confortável, o mais rápido possível. O cronômetro é parado no momento em que o avaliado está em pé. São feitas três tentativas e o melhor valor é considerado.

Tanto a reprodutibilidade como a objetividade do teste de velocidade para levantar-se de uma posição sentada foram altos e significativos $(0,83: \mathrm{p}<0,01[\mathrm{n}=42]$ e 0,86 : $\mathrm{p}$ $<0,01$ [ $n=40]$, respectivamente). O nível de associação com o teste de agilidade (shuttle-run ${ }^{21}$ ) também foi alto e significativo $(0,88)$; no entanto, baixo $(0,12)$ para os testes de flexibilidade (sentar e alcançar ${ }^{22}$ ) e de força muscular (uma repetição máxima ${ }^{23}$ ) no exercício leg press $45^{\circ}$.

Velocidade para levantar-se de uma posição deitada (VLPD). Procedimento semelhante ao teste anterior. O sujeito posiciona-se em decúbito dorsal sobre um colchonete de ginástica; as pernas permanecem encostadas uma à outra; os braços cruzados com o terceiro dedo da mão em direção ao acrômio. $\mathrm{O}$ avaliador fica ao lado direito do avaliado e através da voz de comando "Atenção! Já!" inicia o teste, acionando concomitantemente o cronômetro. $\mathrm{O}$ avaliado deve levantar-se da maneira que lhe for mais confortável, o mais rápido possível. O cronômetro é parado no momento em que o avaliado está em pé. São feitas três tentativas e o melhor valor é considerado.

Os dados de reprodutibilidade e objetividade do teste para levantar-se de uma posição deitada foram significativamente altos e muito altos, 0,71 e 0,99 ( $<<0,01: \mathrm{n}=10)$, respectivamente. A correlação dos valores do teste com os dados de agilidade foi significativa e moderada $(0,48: p<$ $0,01)$, mas fraca e não significativa tanto para o teste sentar e alcançar $(0,10: p>0,01)$ como para o teste de uma repetição máxima $(0,17: \mathrm{p}>0,01)$ no exercício leg press $45^{\circ}$.

Velocidade para calçar e amarrar o tênis (VCAT). O sujeito permanece sentado sobre uma cadeira de modo que a planta dos pés fique totalmente posicionada no solo; os joelhos e os tornozelos afastados lateralmente em distância biacromial; as mãos do sujeito permanecem sobre os respectivos joelhos e cada calçado correspondentemente ao lado externo de cada pé. O avaliador fica em frente do avaliado e através da voz de comando "Atenção! Já!" inicia o teste, acionando concomitantemente o cronômetro. $\mathrm{O}$ avaliado deve calçar e amarrar o tênis como o faz no cotidiano no menor tempo possível. O cronômetro é parado quando o indivíduo acaba de amarrar o último calçado. É feita apenas uma tentativa.
Os resultados iniciais demonstraram reprodutibilidade alta e significativa $(0,83: p<0,01)$, enquanto os valores de objetividade também foram altos e significativos $(0,86$ : $\mathrm{p}$ $<0,01)$. As mesmas características foram observadas na correlação dos resultados do teste de calçar e amarrar o tênis com os de agilidade, sendo de 0,81 ( $\mathrm{p}<0,01: \mathrm{n}=20)$. Em contraste, tanto para o teste de sentar e alcançar como para o de uma repetição máxima no exercício leg press $45^{\circ}$, o nível de associação foi baixo e não significativo $(0,20$ : $\mathrm{p}>0,01 ; \mathrm{n}=20)$.

Velocidade para subir escada (VSE). O sujeito posiciona-se em pé, imediatamente antes do 1 o degrau. Deve subir a escada, que tem 18 degraus ( $h=16 \mathrm{~cm} /$ degrau) e corrimão, na máxima velocidade possível de caminhada. O avaliador fica no local de chegada e através da voz de comando "Atenção! Já!" inicia o teste, acionando concomitantemente o cronômetro. $\mathrm{O}$ avaliado deve subir a escada o mais rápido possível (não podendo correr, mas sim caminhar na máxima velocidade). São utilizados três níveis para determinar a dependência do uso do corrimão para o avaliado subir a escada; 1) Sem Ajuda, o sujeito não necessita do auxílio do corrimão para subir a escada; 2) Ajuda Parcial, necessita do auxílio do corrimão em determinados momentos do teste; e 3) Com Ajuda, o avaliado necessita do auxílio do corrimão durante toda a execução do teste. O cronômetro é parado no momento em que o avaliado ultrapassa o último degrau da escada. São feitas três tentativas com intervalo de dois minutos entre as mesmas; e o melhor valor é considerado.

Foram encontrados valores altos e significativos tanto para a reprodutibilidade como para a objetividade $(0,83 \mathrm{e}$ 0,86: $\mathrm{p}<0,01$; respectivamente). De modo semelhante ao que ocorreu com os demais testes, a correlação com o teste de agilidade foi alta e significativa $(0,89$ : $p<0,01)$; no entanto, baixa para o teste sentar e alcançar $(0,33: p>0,01)$ e fraca para o teste de uma repetição máxima no exercício leg press $45^{\circ}(0,08: \mathrm{p}>0,01)$.

\section{Análise estatística}

A estatística descritiva (medida de tendência central [média aritmética] e de dispersão [desvio padrão]) foi calculada para todas as variáveis contínuas (por exemplo, as medidas de capacidade funcional) de acordo com as variáveis qualitativas (os quartis para cada variável independente) para interpretar os resultados coletados.

Foi calculado o quartil para todas as variáveis independentes que poderiam influenciar na capacidade funcional das voluntárias em realizar as atividades da vida diária. Para tanto, os valores referentes à idade cronológica, peso corporal, altura total, índice de massa corporal, adiposidade total, adiposidade central e adiposidade periférica fo- 
ram divididos em quatro grupos de acordo com o respectivo quartil $(Q)$ de cada variável, que seguiu este modelo: $Q_{1}$ $\left(\mathrm{P}_{0}-\mathrm{P}_{25}\right) ; Q_{2}\left(\mathrm{P}_{25}-\mathrm{P}_{50}\right) ; Q_{3}\left(\mathrm{P}_{50}-\mathrm{P}_{75}\right)$; e $Q_{4}\left(\mathrm{P}_{75}-\mathrm{P}_{100}\right)$. Portanto, os respectivos intervalos para cada variável que são descritos na tabela 3 seguem o modelo descrito acima.

A análise de variância do tipo one way foi empregada para comparar as médias interquartis $\left(Q_{1} \times Q_{2} \times Q_{3} \times Q_{4}\right)$ dos testes VLPS, VLPD, VCAT e VSE para determinada variável independente (idade, peso, altura, IMC, Adp T, Adp C, Adp P) e o post-hoc do tipo Bonferroni foi usado para localizar as possíveis diferenças estatisticamente significativas. O coeficiente de correlação linear de Pearson foi utilizado para verificar o nível de associação entre os testes de predição da capacidade funcional (VLPS, VLPD, VCAT e VSE) para realizar as atividades da vida diária e as variáveis independentes (idade, peso, altura, IMC, Adp T, Adp C, Adp P). Nesse caso, também foi calculado o coeficiente de correlação de Spearman rank-order entre VSE e as variáveis independentes (idade, peso, altura, IMC, Adp T, Adp $\mathrm{C}$, Adp P) devido ao menor número de voluntárias que executaram este teste quando comparado com os demais testes (VLPS, VLPD, VCAT). O intervalo de confiança (IC 95\%) representa a amplitude numérica em que os resultados são prováveis de ocorrer repetindo o experimento e mantendo as mesmas condições. Foi adotado arbitrariamente o nível de significância de $\mathrm{p}<0,05$ para a comparação das médias, enquanto o de $\mathrm{p}<0,01$ para a correlação. O software Statistical Package for the Social Sciences (SPSS: versão 10.0) foi empregado para os cálculos.

\section{RESULTADOS}

A tabela 1 apresenta os resultados descritivos (média \pm desvio padrão, valores mínimo e máximo, e intervalo de confiança [95\%]) referentes à idade cronológica e às características antropométricas das voluntárias.

Dados coletados em outra população pertencente à mesma região geográfica demonstraram valores similares de peso corporal $(69,7 \mathrm{~kg})$, altura $(157,5 \mathrm{~cm})$ e índice de massa corporal $\left(28,3 \mathrm{~kg} / \mathrm{m}^{2}\right)$, independente do nível de atividade física ${ }^{24}$. Não foram encontrados dados que possibilitassem comparação adequada para as variáveis referentes à adiposidade corporal.

Os dados referentes à capacidade funcional para realizar as atividades da vida diária também foram similares aos de

TABELA 1

Características gerais das voluntárias

\begin{tabular}{|c|c|c|c|c|c|}
\hline & \multirow[b]{2}{*}{ Média \pm DPt } & \multirow[b]{2}{*}{ Mínimo } & \multirow[b]{2}{*}{ Máximo } & \multicolumn{2}{|c|}{ IC 95\% } \\
\hline & & & & I & $\mathbf{S}$ \\
\hline Idade (anos) & $63,98 \pm 5,40$ & 47,00 & 77,00 & 63,05 & 64,92 \\
\hline Peso (kg) & $67,20 \pm 9,86$ & 46,50 & 95,10 & 65,50 & 68,91 \\
\hline Altura (cm) & $156,29 \pm 6,11$ & 142,36 & 178,00 & 155,23 & 157,34 \\
\hline IMC (kg/m²) & $27,55 \pm 4,05$ & 18,53 & 39,60 & 26,85 & 28,25 \\
\hline $\operatorname{Adp} T(m m)$ & $201,90 \pm 51,93$ & 85,96 & 348,90 & 192,93 & 210,88 \\
\hline Adp C (mm) & $103,16 \pm 28,65$ & 33,57 & 180,43 & 98,21 & 108,11 \\
\hline Adp P (mm) & $98,74 \pm 29,55$ & 32,63 & 205,00 & 93,63 & 103,85 \\
\hline
\end{tabular}

† Os valores referem-se a média \pm desvio padrão; ₹ IC 95\% = I (valor inferior) e S (valor superior)

TABELA 2

Valores de capacidade funcional para realizar as atividades da vida diária

\begin{tabular}{lrrrrr}
\hline & & & & \multicolumn{2}{c}{ IC 95\%⿱ } \\
\cline { 4 - 6 } & Média \pm DPt & Mínimo & Máximo & I & S \\
& & & & & \\
VLPS (seg.) & $3,42 \pm 1,08$ & 1,84 & 7,49 & 3,21 & 3,59 \\
VLPD (seg.) & $4,04 \pm 1,10$ & 2,40 & 9,07 & 3,82 & 4,21 \\
VCAT (seg.) & $27,07 \pm 8,38$ & 14,24 & 73,78 & 25,60 & 28,65 \\
VSE (seg.) & $8,00 \pm 1,18$ & 6,37 & 10,67 & 7,63 & 8,36 \\
\hline
\end{tabular}

† Os valores referem-se a média \pm desvio padrão; $¥$ IC $95 \%$ =I (valor inferior) e S (valor superior). 
outro estudo que submeteu mulheres na faixa etária de 55 a 85 anos a um programa de exercício aeróbico e outro de exercícios com pesos, mesmo quando foi considerado o efeito de ambos os protocolos de treinamento nessas variáveis ${ }^{25}$.

A tabela 3 apresenta os valores absolutos referentes à performance nos testes de capacidade funcional para realizar as atividades da vida diária (VLPS, VLPD, VCAT, VSE) de acordo com as variáveis independentes (idade, peso, altura, IMC, Adp T, Adp C, Adp P) divididas por quartil.

Com exceção do teste velocidade para calçar e amarrar o tênis (VCAT), todos os demais testes (VLPS, VLPD, VSE) apresentaram diferenças estatisticamente significativas ( $p$ $<0,05$ ) interquartis quando a idade cronológica foi considerada. A idade cronológica foi a única variável independente capaz de produzir diferenças estatisticamente signi-

TABELA 3

Valores ${ }^{\dagger}$ de capacidade funcional para realizar as atividades da vida diária de acordo com as variáveis independentes divididas por quartil

\begin{tabular}{|c|c|c|c|c|}
\hline & VLPS & VLPD & VCAT & VSE \\
\hline \multicolumn{5}{|l|}{ Idade (anos) } \\
\hline$Q_{1}:<60$ & $3,11 \pm 0,80(28)$ & $3,68 \pm 1,08$ (28) & $25,90 \pm 8,99(28)$ & $7,57 \pm 0,64(8)$ \\
\hline$Q_{2}^{1}: 60-63$ & $3,13 \pm 0,86(28)$ & $3,71 \pm 0,80(28)$ & $24,59 \pm 5,31(29)$ & $7,43 \pm 1,28(9)$ \\
\hline$Q_{3}: 63-67$ & $3,38 \pm 1,16(28)$ & $3,98 \pm 1,02(30)$ & $29,24 \pm 10,82(29)$ & $7,71 \pm 0,70(9)$ \\
\hline$Q_{4}: \geq 67$ & $3,85 \pm 1,20^{1,2 \ddagger}(42)$ & $4,54 \pm 1,19^{1,2,3}(42)$ & $28,05 \pm 7,48(43)$ & $8,69 \pm 1,27^{2}(16)$ \\
\hline \multicolumn{5}{|l|}{ Peso (kg) } \\
\hline$Q_{1}:<59$ & $2,99 \pm 0,68(31)$ & $3,75 \pm 0,88(31)$ & $26,13 \pm 5,34(32)$ & $7,54 \pm 0,97(10)$ \\
\hline$Q_{2}: 59,9-66,5$ & $3,22 \pm 1,05(32)$ & $3,91 \pm 1,04(32)$ & $26,97 \pm 10,10(32)$ & $7,53 \pm 0,64(12)$ \\
\hline$Q_{3}: 66,5-74,10$ & $3,71 \pm 1,25(30)$ & $4,21 \pm 1,21(30)$ & $26,37 \pm 6,21(33)$ & $8,64 \pm 1,37(8)$ \\
\hline$Q_{4}: \geq 74,10$ & $3,76 \pm 1,11^{1}(33)$ & $4,28 \pm 1,20(33)$ & $28,85 \pm 10,74(32)$ & $8,42 \pm 1,36(12)$ \\
\hline \multicolumn{5}{|l|}{ Altura (cm) } \\
\hline$Q_{1}:<151,90$ & $3,41 \pm 1,11(31)$ & $3,92 \pm 0,92(31)$ & $25,83 \pm 5,49(31)$ & $7,76 \pm 0,50(10)$ \\
\hline$Q_{2}: 151,90-155,70$ & $3,42 \pm 1,08(32)$ & $4,16 \pm 1,31(32)$ & $27,98 \pm 8,15(33)$ & $7,82 \pm 1,04(11)$ \\
\hline$Q_{3}: 155,70-160,80$ & $3,54 \pm 1,19(32)$ & $4,11 \pm 1,13(32)$ & $26,70 \pm 6,50(32)$ & $8,52 \pm 1,82(7)$ \\
\hline$Q_{4}: \geq 160,80$ & $3,32 \pm 0,96(31)$ & $3,96 \pm 1,03(31)$ & $27,71 \pm 11,94(33)$ & $8,05 \pm 1,27(14)$ \\
\hline \multicolumn{5}{|l|}{ IMC (kg/ m²) } \\
\hline$Q_{1}:<24,69$ & $2,99 \pm 0,66(31)$ & $3,66 \pm 0,61(31)$ & $26,30 \pm 7,85(32)$ & $7,57 \pm 0,96(12)$ \\
\hline$Q_{2}: 24,69-27,29$ & $3,25 \pm 0,94(33)$ & $4,04 \pm 1,17(33)$ & $26,63 \pm 8,38(32)$ & $8,15 \pm 1,19(13)$ \\
\hline$Q_{3}: 27,29-29,60$ & $3,59 \pm 1,21(30)$ & $4,15 \pm 1,26(30)$ & $25,91 \pm 6,18(33)$ & $7,61 \pm 0,86(8)$ \\
\hline$Q_{4}: \geq 29,60$ & $3,86 \pm 1,25^{1}(32)$ & $4,31 \pm 1,19(32)$ & $29,49 \pm 10,52(32)$ & $8,70 \pm 1,43(9)$ \\
\hline \multicolumn{5}{|l|}{ Adp T (mm) } \\
\hline$Q_{1}:<165,43$ & $3,08 \pm 0,83(31)$ & $3,67 \pm 0,79(31)$ & $25,30 \pm 6,53(32)$ & $7,45 \pm 1,16(7)$ \\
\hline$Q_{2}: 165,43-200,64$ & $3,05 \pm 0,79(32)$ & $3,83 \pm 0,67(32)$ & $26,01 \pm 8,91$ & $7,89 \pm 0,91(12)$ \\
\hline$Q_{3}: 200,64-236,67$ & $3,40 \pm 1,00(31)$ & $4,00 \pm 1,18(31)$ & $26,83 \pm 6,10(32)$ & $7,93 \pm 1,12(9)$ \\
\hline$Q_{4}: \geq 236,67$ & $4,15 \pm 1,27^{1,2,3}(32)$ & $4,64 \pm 1,39^{1,2}(32)$ & $30,18 \pm 10,68(32)$ & $8,41 \pm 1,38(14)$ \\
\hline \multicolumn{5}{|l|}{ Adp C (mm) } \\
\hline$Q_{1}:<82,33$ & $3,12 \pm 0,87(31)$ & $3,77 \pm 0,99(31)$ & $26,64 \pm 8,12(32)$ & $7,64 \pm 1,20(8)$ \\
\hline$Q_{2}: 82,33-104,14$ & $3,07 \pm 0,66(31)$ & $3,72 \pm 0,66(31)$ & $25,81 \pm 7,45$ & $7,85 \pm 0,92(11)$ \\
\hline$Q_{3}: 104,14-119,67$ & $3,32 \pm 1,00(32)$ & $3,97 \pm 0,97(32)$ & $26,05 \pm 6,31(33)$ & $7,93 \pm 1,13(9)$ \\
\hline$Q_{4}: \geq 119,67$ & $4,15 \pm 1,33^{1,2,3}(32)$ & $4,68 \pm 1,40^{1,2,3}(32)$ & $29,96 \pm 10,88(31)$ & $8,36 \pm 1,38(14)$ \\
\hline \multicolumn{5}{|l|}{ Adp P (mm) } \\
\hline$Q_{1}:<78,27$ & $2,98 \pm 0,88(31)$ & $3,70 \pm 0,81(31)$ & $26,90 \pm 8,01(32)$ & $7,38 \pm 1,18(6)$ \\
\hline$Q_{2}: 78,27-96,42$ & $3,13 \pm 0,73(33)$ & $3,77 \pm 0,74(33)$ & $25,70 \pm 7,28(32)$ & $7,67 \pm 0,58(9)$ \\
\hline$Q_{3}: 96,42-117,97$ & $3,79 \pm 1,40^{1}(30)$ & $4,22 \pm 1,38(30)$ & $25,60 \pm 7,34(32)$ & $7,97 \pm 0,99(14)$ \\
\hline$Q_{4}: \geq 117,97$ & $3,81 \pm 0,99^{1,2}(32)$ & $4,48 \pm 1,22^{1}(32)$ & $30,01 \pm 10,12(33)$ & $8,55 \pm 1,50(13)$ \\
\hline
\end{tabular}

† Os valores referem-se a média \pm desvio padrão (total de voluntárias por quartil e teste) na unidade de tempo (em segundos); $\ddagger$ Os números em sobrescrito $(1,2,3,4)$ inseridos na tabela representam as diferenças estatisticamente significativas interquartis (Q1, Q2, Q3, Q4) para as respectivas variáveis. 
ficativas interquartis no teste VSE $\left(Q_{2} \times Q_{4}\right)$ (tabela 3). É bastante provável que essa diferença represente principalmente a performance per se do que especificamente o uso associado do corrimão como dependência para a execução do teste. Nenhuma das voluntárias necessitou do auxílio do corrimão durante o teste $\left(Q_{4}\right)$.

De acordo com a tabela 3, o peso corporal assim como o índice de massa corporal foram as variáveis independentes que menor repercussão induziram sobre a performance nos testes de capacidade funcional para realizar as atividades da vida diária entre as variáveis que alcançaram significância estatística. Ambas as variáveis independentes exerceram efeito negativo no VLPS quando os quartis das extremidades foram comparados $\left(Q_{1} \times Q_{4}\right)$.

A altura total exerceu efeito nulo sobre a capacidade funcional para realizar as atividades da vida diária, independente do teste analisado (VLPS, VLPD, VCAT, VSE), conforme pode ser observado na tabela 3 . Isso sugere que essa variável não induz efeito negativo na capacidade funcional de mulheres acima de 47 anos de idade e/ou que seja necessário maior número de voluntárias para determinar qualquer tipo de influência.

A adiposidade corporal representou a variável independente que maior efeito negativo exerceu sobre a capacidade funcional para realizar as atividades da vida diária, independente da distribuição de gordura corporal (central ou periférica). A adiposidade central seguida da adiposidade total e periférica foi a variável mais influente (tabela 3 ).

Os dados referentes às variações percentuais interquartis para determinado teste de capacidade funcional para realizar as atividades da vida diária de acordo com as variáveis independentes que alcançaram significância estatística são apresentados na tabela 4. A magnitude de variação percentual ocorreu entre a amplitude de $14,1 \%$ (idade [VLPD: $Q_{3} \times Q_{4}$ ]) e $36,1 \%$ (Adp T [VLPS: $Q_{3} \times Q_{4}$ ]). Em ordem, as variáveis independentes que exerceram maior repercussão negativa sobre a performance nos testes de capacidade funcional (VLPS, VLPD, VSE) para realizar as atividades da vida diária foram Adp C, idade, Adp T, Adp $\mathrm{P}$, IMC, e peso.

As maiores diferenças percentuais interquartis ocorreram entre os quartis localizados nas extremidades $\left(Q_{1} \mathrm{e}\right.$ $Q_{4}$ ), com exceção do teste VLPS, que apresentou variação percentual discretamente superior quando os quartis $Q_{2} \mathrm{e}$ $Q_{4}$ foram comparados.

Foram verificados níveis de associação estatisticamente significativos que variaram de 0,21 (Adp C x VCAT) a 0,40 (Idade $\mathrm{x}$ VSE) quando as variáveis independentes foram correlacionadas com os testes de predição da capacidade funcional (tabela 5). A altura foi a única variável independente que não apresentou correlação estatisticamente significativa com nenhum teste de predição da capacidade funcional para realizar as atividades da vida diária. Por outro lado, com exceção da Adp C $(0,21: p<0,01)$, o teste VCAT não se correlacionou com nenhuma das variáveis analisadas neste estudo, sugerindo que estas variáveis não influenciaram a performance deste teste e/ou que outras variáveis devem ser consideradas.

Não houve correlação estatisticamente significativa entre as demais variáveis independentes e o VSE (exceção para idade $[0,40]$, peso $[0,38]$ e adiposidade periférica $[0,31])$, embora o ponto de corte para significância estatística tenha sido similar ao apresentado nas correlações dos testes VLPS e VLPD com as variáveis IMC, Adp T e Adp C (variação de 0,23 a 0,39: p < 0,01). É possível que o número de voluntárias submetidas ao VSE tenha sido insuficiente para produzir associação significativa - com as variáveis independentes IMC, Adp T e Adp C - nessa magnitude de fenômeno; todavia, essa característica foi similar, independente do tratamento estatístico empregado (Pearson ou Spearman). Paradoxalmente, é importante ressaltar que o nível de associação entre VSE e altura foi, no mínimo, cerca de quatro vezes superior $(0,25)$ quando comparado com o alcançado entre essa mesma variável independente e VLPS $(-0,01)$, VLPD $(0,03)$ e VCAT $(0,06)$ (tabela 5$)$.

TABELA 4

Variação percentual $(\Delta \%)$ interquartil para as variáveis que alcançaram significância estatística

\begin{tabular}{|c|c|c|c|c|c|c|c|c|}
\hline & \multicolumn{4}{|c|}{ VLPS } & \multicolumn{3}{|c|}{ VLPD } & \multirow{2}{*}{$\frac{\text { VSE }}{Q_{2} \times Q_{4}}$} \\
\hline & $Q_{1} \times Q_{3}$ & $Q_{1} \times Q_{4}$ & $Q_{2} \times Q_{4}$ & $Q_{3} \times Q_{4}$ & $Q_{1} \times Q_{4}$ & $Q_{2} \times Q_{4}$ & $Q_{3} \times Q_{4}$ & \\
\hline Idade & - & 23,8 & 23,0 & - & 23,4 & 22,4 & 14,1 & 17,0 \\
\hline Peso & - & 25,7 & - & - & - & - & - & - \\
\hline IMC & - & 29,1 & - & - & - & - & - & - \\
\hline Adp T & - & 34,7 & 36,1 & 22,0 & 26,4 & 21,1 & - & - \\
\hline Adp C & - & 33,0 & 35,2 & 25,0 & 24,1 & 25,8 & 17,9 & - \\
\hline Adp P & 27,2 & 27,8 & 21,7 & - & 21,1 & - & - & - \\
\hline
\end{tabular}




\begin{tabular}{|c|c|c|c|c|c|c|c|c|c|c|c|}
\hline \multicolumn{12}{|c|}{$\begin{array}{l}\text { TABELA } 5 \\
\text { Matriz de correlação entre as variáveis independentes e os testes de } \\
\text { predição da capacidade funcional para realizar as atividades da vida diária }\end{array}$} \\
\hline & Idade & Peso & Altura & IMC & Adp T & Adp C & Adp P & VLPS & VLPD & VCAT & VSE $^{\dagger}$ \\
\hline Idade & 1,00 & & & & & & & & & & \\
\hline Peso & $-0,11$ & 1,00 & & & & & & & & & \\
\hline Altura & $-0,04$ & $0,27^{\ddagger}$ & 1,00 & & & & & & & & \\
\hline IMC & $-0,09$ & $0,86^{\ddagger}$ & $-0,26^{\ddagger}$ & 1,00 & & & & & & & \\
\hline Adp T & $-0,05$ & $0,74^{\ddagger}$ & $-0,02$ & $0,75^{\ddagger}$ & 1,00 & & & & & & \\
\hline Adp C & $-0,05$ & $0,69^{\ddagger}$ & $-0,02$ & $0,70^{\ddagger}$ & $0,89^{\ddagger}$ & 1,00 & & & & & \\
\hline Adp P & $-0,05$ & $0,63^{\ddagger}$ & $-0,02$ & $0,64^{\ddagger}$ & $0,90^{\ddagger}$ & $0,59^{\ddagger}$ & 1,00 & & & & \\
\hline VLPS & $0,32^{\ddagger}$ & $0,34^{\ddagger}$ & $-0,01$ & $0,35^{\ddagger}$ & $0,39^{\ddagger}$ & $0,36^{\ddagger}$ & $0,33^{\ddagger}$ & 1,00 & & & \\
\hline VLPD & $0,34^{\ddagger}$ & $0,25^{\ddagger}$ & 0,03 & $0,23^{\ddagger}$ & $0,34^{\ddagger}$ & $0,33^{\ddagger}$ & $0,27^{\ddagger}$ & $0,79^{\ddagger}$ & 1,00 & & \\
\hline VCAT & 0,11 & 0,15 & 0,06 & 0,12 & 0,17 & $0,21^{\ddagger}$ & 0,09 & $0,37^{\ddagger}$ & $0,36^{\ddagger}$ & 1,00 & \\
\hline VSE $^{1}$ & $0,40^{\ddagger}$ & $0,38^{\ddagger}$ & 0,25 & 0,24 & 0,30 & 0,26 & $0,31^{\ddagger}$ & $0,55^{\ddagger}$ & $0,51^{\ddagger}$ & 0,05 & 1,00 \\
\hline
\end{tabular}

† Foram utilizadas 42 voluntárias para a correlação entre as variáveis independentes e o VSE; $¥ p<0,01$.

\section{DISCUSSÃO}

Os dados deste estudo fornecem evidências claras de que a adiposidade corporal total e as suas distribuições (central e periférica) assim como a idade cronológica foram as principais variáveis independentes que exerceram efeito negativo na capacidade funcional para realizar as atividades da vida diária de mulheres acima de 47 anos. A adiposidade corporal total exerceu efeitos negativos na capacidade funcional que variaram em magnitude de $21,1 \%$ (VLPD) a $36,1 \%$ (VLPS), enquanto a adiposidade corporal central e periférica explicaram, respectivamente, cerca de 35,2\% (VLPS) e $27,8 \%$ (VLPS) do decréscimo da capacidade das voluntárias em realizar as atividades da vida diária. Isso, em geral, significa que o excesso de adiposidade corporal (total, central ou periférica) explica cerca de $30 \%$ da limitação na performance em testes de capacidade funcional, embora seja necessária análise estatística mais refinada para poder seguramente corroborar esta alusão independentemente.

Por outro lado, além dos testes VLPS e VLPD, a idade cronológica foi a única variável que demonstrou influenciar negativamente a performance no teste VSE (tabela 3 ). A idade de 67 anos ou mais representou cerca de $17 \%$ de decréscimo no rendimento desse teste (tabela 4), enquanto a amplitude etária de 47 a 77 anos de idade explica aproximadamente $16 \%$ do declínio no rendimento durante o VSE (VSE $x$ idade: $r=0,40\left[r^{2}=0,16\right]$ ) (tabela 4).

Esses dados reforçam achados anteriores de que o elevado valor do IMC é um dos fatores predisponentes à incapacidade funcional ${ }^{26-29}$, mas também demonstram a limitação parcial tanto do peso corporal como do índice de massa corporal como parâmetros isolados para a determinação da influência destas variáveis sobre a performance em testes de capacidade funcional, no mínimo, em pessoas nessa faixa etária, devido ao fato de que estas duas variáveis não foram sensíveis o suficiente para detectar discreto efeito estatisticamente significativo interquartis quando comparadas com a adiposidade corporal (total, central, periférica) (tabela 3).

Mesmo assim, a análise absoluta feita a partir dos pontos de corte para ambas as variáveis obscurece os valores limiares relativos que exacerbam o problema do excesso de peso. Ou seja, quando as voluntárias são comparadas de acordo com o ponto de corte para cada quartil, é observado que somente aquelas localizadas na região $\geq 74,1 \mathrm{~kg}\left(Q_{4}\right)$ para o peso corporal e nas regiões obesidade e obesidade mórbida ( $\left.\geq 29,6 \mathrm{~kg} / \mathrm{m}^{2}\left[Q_{4}\right]\right)$ para o índice de massa corporal apresentaram decréscimo dos valores no teste VLPS (na comparação com $Q_{1}$ ), sugerindo que, talvez, somente grandes incrementos no peso corporal representem limitações à capacidade funcional. Contudo, de forma conservadora, quando comparada com a mediana, esses valores demonstram que são necessárias, no mínimo, unidades (kg e kg/ $\mathrm{m}^{2}$ ) em excesso, de aproximadamente $11,4 \%$ para o peso corporal e de $8,5 \%$ para o IMC, para que sejam, respectivamente, diagnosticadas limitações na capacidade para levantar-se de uma posição sentada de $25,7 \%$ e de $29,1 \%$. Isso significa que a cada quilograma adicional acima do limiar $\geq 74,1 \mathrm{~kg}$ existe decréscimo de $2,2 \%$ na performance no teste VLPS, enquanto para o IMC a magnitude de fenômeno representa 3,4\% para cada unidade acima de 29,6 $\mathrm{kg} / \mathrm{m}^{2}$.

É importante ressaltar que tanto o peso como o índice de massa corporal apresentaram nível de associação com as 
variáveis adiposidade corporal total, central e periférica, que variaram de moderado (peso x Adp P: 0,63 [p < 0,01]; IMC x Adp P: 0,64 [p < 0,01]) a alto (peso x Adp T: 0,74 [p $<0,01$ ]; IMC x Adp T: 0,75 [p < 0,01]), enquanto que, para a altura, o nível de associação foi baixo, porém significativo $(\mathrm{p}<0,01)$, tanto para o peso $(0,27)$ como para o IMC $(-0,26)$, sendo que, para o último, a correlação apresentou direção inversa (tabela 5). O que demonstra que a adiposidade corporal explicou cerca de $40 \%$ a $55 \%$, seja do peso ou do índice de massa corporal, e que, independente da direção, o poder estatístico da altura em influenciar o peso assim como o índice de massa corporal foi de somente $7 \%$. Isso sugere que existem outras variáveis além daquelas consideradas no presente estudo (por exemplo, massa magra) que estão diretamente associadas a esses dois parâmetros (peso e índice de massa corporal) e, portanto, impedem a análise dos efeitos reais sobre a capacidade funcional em realizar atividades da vida diária quando os valores não são ajustados à maioria das variáveis de confusão.

Surgem alguns pontos interessantes para análise quando somente os valores de correlação intertestes de capacidade funcional são considerados. Houve correlação estatisticamente significativa $(\mathrm{p}<0,01)$ que variou de 0,36 (VLPD $\mathrm{x}$ VCAT) a 0,79 (VLPS x VLPD) entre os testes propostos para medir a capacidade funcional nas atividades da vida diária. A única exceção foi a correlação entre VCAT e VSE, que foi fraca e não significativa $(0,05)$ (tabela 5). Esses dados sugerem que a performance em determinado teste de capacidade funcional pode explicar em até $62 \%$ o rendimento em outro teste de capacidade funcional (no caso, VLPS e VLPD) com exigências biomecânicas similares, mas que mesmo testes que apresentam níveis extremamente diferentes de exigência física (por exemplo, VLPS e VCAT) podem per se, mutuamente, explicar cerca de $14 \%$ dos resultados finais, independente da análise de outras variáveis (adiposidade corporal, força muscular). Embora baixo, esse valor $(14 \%)$ pode representar para o indivíduo dependente agudo ou crônico, residente seja em asilo ou em comunidade, a possibilidade de encurtar a distância entre os limiares de independência e falência funcionais, respectivamente, por meio da simples estratégia de preservar-se ativo nas atividades mais simples da vida diária e, como conseqüência, podendo retardar o surgimento de problemas degenerativos graves associados e comuns à limitação física.

Outra questão que merece descrição refere-se à associação entre idade cronológica e as variáveis antropométricas (exceção para a altura). De modo surpreendente, os dados deste estudo demonstraram existir correlação teoricamente nula entre idade cronológica e peso, IMC, Adp T, Adp C ou Adp P. Os valores de correlação foram todos fracos e não significativos, variando de $-0,05$ (idade x Adp) a
$-0,11$ (idade x peso) (tabela 5). Isso faz presumir que, provavelmente, a idade não explique, pelo menos nessa população, valores elevados de peso, IMC, Adp T, Adp C ou Adp, e que existam variáveis como o nível de atividade física e os hábitos alimentares que poderiam influenciar diretamente os valores referentes a esses parâmetros morfológicos e até mesmo a capacidade funcional, visto que existem dados demonstrando que os parâmetros nutricionais e a composição corporal predizem, em um período de dois anos, a progressão da incapacidade funcional em pessoas idosas ${ }^{30}$.

Em estudo anterior ${ }^{5}$, foi verificada relação inversamente proporcional entre adiposidade corporal total (média de três dobras cutâneas [tríceps, subescapular e suprailíaca]) e performance em testes de aptidão física (agilidade e impulsão horizontal) em 53 mulheres na faixa etária de 49 a 82 anos de idade. Foi observado que o grupo de voluntárias localizado acima da média para a adiposidade corporal ( $\geq 1$ desvio padrão [DP]) apresentava estatisticamente menores resultados nos testes de agilidade e impulsão horizontal quando comparado com as voluntárias localizadas, seja na média (<-1DP a < 1DP) (impulsão horizontal: $\mathrm{p}<0,05)$ ou abaixo da média ( $\leq-1 \mathrm{DP}$ ) (impulsão horizontal e agilidade: $p<0,0001$ ). A variação percentual representou cerca de $29,4 \%$ para a agilidade e $66,9 \%$ para a impulsão horizontal quando comparados os grupos abaixo e acima da média, e $17,4 \%$ para a agilidade e $19,7 \%$ para a impulsão horizontal, na comparação entre os grupos dentro da média e acima da média.

Zamboni et al. ${ }^{29}$ demonstraram que o peso corporal, IMC, relação cintura-quadril, massa total de gordura, massa de gordura dos membros inferiores e a percentagem de gordura corporal foram significativamente superiores em mulheres idosas que apresentavam incapacidade funcional (determinada por meio de três escalas para as atividades da vida diária e atividades instrumentais da vida diária, e testes de capacidade funcional [por exemplo, caminhar $800 \mathrm{~m}$ e subir escadas]) do que em mulheres que não exibiam limitação funcional. No entanto, quando os dados foram ajustados para a presença de doenças, essas diferenças desapareceram (exceção para massa gorda total e massa gorda de membros inferiores).

Ricardo e Araújo ${ }^{7}$ submeteram um grupo de 461 pessoas de ambos os sexos e na faixa etária entre 18 a 88 anos de idade ao teste sentar-levantar (TSL) proposto por Araú$\mathrm{jo}^{4}$. Os autores empregaram três diferentes métodos de relação peso para a altura (IMC, recíproco do índice ponderal [altura/peso ${ }^{1 / 3}$ : RIP] e o componente ectomórfico adimensional do somatótipo de Heath-Carter [ECTO]) para analisar o efeito médio assim como o efeito do excesso de peso representado por cada parâmetro (IMC: $>25 \mathrm{~kg} / \mathrm{m}^{2}$; RIP: 
$<41 \mathrm{~cm} / \mathrm{kg}^{1 / 3}$; ЕСTO: < 1,45) na performance do TSL. Foi verificado que os sujeitos que necessitavam de algum tipo de apoio para sentar-se ou levantar-se (TSL $<5 / 5)$ apresentavam estatisticamente piores resultados $(\mathrm{p}<0,05)$ em todos os parâmetros de relação de peso para a altura (IMC, RIP, ECTO) quando comparados com os sujeitos que não precisavam de nenhum tipo de apoio $(\mathrm{TSL}=5 / 5)$, independente do sexo. Quando os sujeitos foram ajustados aos pontos de corte para os diferentes parâmetros, foi verificada tendência não significativa de maior necessidade de apoio durante o TSL naqueles que se localizavam no grupo excesso de peso em comparação com os grupos abaixo do peso e região normal para ambos os sexos. Todavia, a magnitude do fenômeno foi mais que duas vezes maior para o sexo feminino $(-20,4 \%)$ do que para o masculino $(-9,1 \%)$. Também foi demonstrado por meio dos coeficientes de correlação que tanto o RIP como o ECTO têm influência negativa discretamente maior sobre o ato de sentar-se (RIP e ECTO: 0,26 [masculino]; RIP e ECTO: 0,59 [feminino]) do que sobre o ato de levantar-se (RIP e ECTO: 0,30 [masculino]; RIP e ECTO: 0,63 [feminino]).

A limitação funcional pode também variar de acordo com o ponto de corte utilizado para determinado critério. Por exemplo, Friedman et al. ${ }^{31}$ verificaram que mulheres, mas não homens, localizadas no quintil superior para o IMC tinham maior risco de dependência funcional, mas quando o IMC foi classificado de acordo com as normas estabelecidas pelo National Institute of Health (NIH), tanto homens como mulheres localizados no IMC maior que $40 \mathrm{~kg} / \mathrm{m}^{2}$ tinham significativamente maior risco de dependência funcional para as atividades da vida diária (AVD) assim como para as atividades instrumentais da vida diária (AIVD) autoreportadas.

Por outro lado, existem estudos documentando que o decréscimo do peso corporal, ao ponto em que se pode considerar como um dos marcadores de desnutrição, também tem efeito negativo na capacidade funcional. Tully e Snowdon ${ }^{32}$ acompanharam uma coorte de 475 mulheres (75-99 anos) de congregações religiosas durante dois anos e observaram que o risco de dependência em atividades como tomar banho, caminhar, vestir-se, comer, levantar-se e usar o toalete era 2,5 vezes maior no grupo de pessoas que apresentou decréscimo no peso corporal superior a $3 \%$ durante o período analisado. Outro estudo verificou que o risco de dependência funcional e mortalidade em 210 pessoas (acima de 65 anos e de ambos os sexos) que viviam na comunidade era maior entre os indivíduos que se posicionavam no IMC menor que $22 \mathrm{~kg} / \mathrm{m}^{2}$ quando comparados com os valores de IMC 22 a $27 \mathrm{~kg} / \mathrm{m}^{2}$ ou maior que $27 \mathrm{~kg} /$ $\mathrm{m}^{2}$. No caso do último, não houve correlação significativa com o risco de mortalidade ${ }^{33}$, sugerindo que valores extre- mos de composição corporal são, independente da direção, fatores predisponentes à incapacidade funcional, assim como mortalidade em pessoas idosas.

\section{CONCLUSÃO}

Os resultados deste estudo suportam a hipótese de que a adiposidade corporal e a idade cronológica estão entre as principais variáveis independentes que induzem efeitos negativos sobre a capacidade de mulheres de 47 anos ou mais em realizar as atividades da vida diária. Isso reforça a necessidade de estratégias como a adoção de um estilo de vida fisicamente ativo, assim como a de hábitos alimentares saudáveis ao longo da vida e também durante a idade avançada.

Há de ressaltar que tais condutas devem priorizar especialmente o gerenciamento da deposição local e total de gordura, visto que, não necessariamente, o excesso de peso corporal e/ou o índice de massa corporal elevado refletiram decréscimo significativo na capacidade funcional.

De qualquer modo, o acompanhamento ao longo dos anos e a realização de estudos futuros que utilizem tratamento estatístico que permita quantificar o real efeito de cada variável independente sobre a capacidade funcional para realizar as atividades da vida diária (como, por exemplo, a análise de regressão logística), possibilitarão a compreensão melhor das variáveis estudadas. A magnitude com que essas variáveis influenciam a capacidade funcional para realizar as atividades da vida diária também deverá ser mais explorada.

\section{AGRADECIMENTOS}

$\mathrm{O}$ autor expressa seus agradecimentos à Secretaria Municipal de Esportes e Turismo de Americana (São Paulo), assim como à Academia Modulus (unidade Americana) por subsidiarem parcialmente as três fases do Estudo do Envelhecimento de Americana. Também estende os agradecimentos aos Profs. Ddos. Alex Antonio Florindo (Faculdade de Saúde Pública - USP) e Raul Santo de Oliveira (Escola Paulista de Medicina - Unifesp) por auxiliarem na coleta dos dados.

\section{REFERÊNCIAS}

1. Ostchega Y, Harris TB, Hirsch R, Parsons VL, Kington R, Katzoff M. Reliability and prevalence of physical performance examination assessing mobility and balance in older persons in the US: data from the Third National Health and Nutrition Examination Survey. J Am Geriatr Soc 2000;48:1136-41.

2. Ostchega Y, Harris TB, Hirsch R, Parsons VL, Kington R. The prevalence of functional limitations and disability in older persons in the US: data from the National Health and Nutrition Examination Survey III. J Am Geriatr Soc 2000;48:1132-5. 
3. Andreotti RA, Okuma SS. Validação de uma bateria de testes de atividades da vida diária para idosos fisicamente independentes. Rev Paulista Educ Física 1999;13:46-66.

4. Araújo CGS. Teste de sentar-levantar: apresentação de um procedimento para avaliação em Medicina do Exercício e do Esporte. Rev Bras Med Esporte 1999;5:179-82.

5. Raso V, Andrade EL, Matsudo SMM, Matsudo VKR. Impacto de la adiposidad corporal sobre la agilidad y fuerza muscular de mujeres ancianas - Proyecto Americana. Anales del XVIII Congreso Panamericano de Medicina Deportiva, Colombia 1999;175.

6. Lira VA, Silva EB, Araújo CGS. As ações de sentar e levantar do solo são prejudicadas por excesso de peso. Rev Bras Med Esporte 2000;6: 241-8.

7. Ricardo DR, Araújo CGS. Teste de sentar-levantar: influência do excesso de peso corporal em adultos. Rev Bras Med Esporte 2001;7:45-52.

8. Nourhashémi F, Andrieau S, Gillette-Guyonnet S, Vellas B, Albarède JL, Grandjean H. Instrumental activities of daily living as a potential marker of frailty: a study of 7364 community-dwelling elderly women (the EPIDOS Study). J Gerontol A Biol Sci Med Sci 2001;56A:M44853.

9. Barberger-Gateau P, Fabrigoule C, Helmer C, Rouch I, Dartigues JF. Functional impairment in instrumental activities of daily living: an early clinical sign of dementia? J Am Geriatr Soc 1999;47:456-62.

10. Markides KS, Black SA, Ostir GV, Angel RJ, Guralnik JM, Lichtenstein M. Lower body function and mortality in Mexican American elderly people. J Gerontol A Biol Sci Med Sci 2001;56A:M243-7.

11. Michael K. Relationship of skeletal muscle atrophy to functional status: a systematic research review. Biol Res Nurs 2000;2:117-31.

12. Ito $\mathrm{H}$, Ohshima $\mathrm{A}$, Ohto $\mathrm{N}$, Ogasawara $\mathrm{M}$, Tsuzuki $\mathrm{M}$, Takao K, et al. Relation between body composition and age in healthy Japanese subjects. Eur J Clin Nutr 2001;55:462-70.

13. DeNino WF, Tchernof A, Dionne IJ, Toth MJ, Ades PA, Sites CK, et al Contribution of abdominal adiposity to age-related differences in insulin sensitivity and plasma lipids in healthy nonobese women. Diabetes Care 2001;24:925-32.

14. Grinker JA, Tucker KL, Vokonas OS, Rush D. Changes in patterns of fatness in adult men in relation to serum indices of cardiovascular risk: the Normative Aging Study. Int J Obes Relat Metab Disord 2000;24: 1369-78.

15. Klein BE, Klein R, Lee KE, Jensen SC. Measures of obesity and agerelated eye diseases. Ophthalmic Epidemiol 2001;8:251-62.

16. Eisman JA. Genetics of osteoporosis. Endocr Rev 1999;20:788-804.

17. Pawelec G, Effros RB, Caruso C, Remarque E, Barnett Y, Solana R. T cells and aging. Front Biosci 1999;4:D216-69.

18. Fried LP, Guralnik JM. Disability in older adults: evidence regarding significance, etiology, and risk. J Am Geriatr Soc 1997;45:92-100.

19. França NM, Vívolo MA. Medidas antropométricas. In: Matsudo VKR, editor. Testes em ciências do esporte. 6a ed. São Caetano do Sul, 1998; 19-32.
20. Harrisson GG, Buskirk ER, Lindsay-Carter JE, Johnston FE, Lohman TG, Pollock ML, et al. Skinfold thicknesses and measurement technique. In: Lohman TG, Roche AF, Martorell R, editors. Anthropometric standardization reference manual. Champaign, IL: Human Kinetics, 1998; 55-70.

21. Stanziola L, Prado JS. Medidas da agilidade. In: Matsudo VKR, editor. Testes em ciências do esporte. 4a ed. São Paulo: Ed. Gráficos Burti, 1998; 73-7.

22. Canadian Ministry of State, Fitness and Amateur Sport. Canadian Standardized Test of Fitness (for 15 to 69 years of age). Published by Authority of the Minister of State, Fitness and Amateur Sport. Operation's Manual. $3^{\text {rd }}$ ed., 1987.

23. Heyward VH. Designing resistance training programs. In: Heyward VH, editor. Advanced fitness assessment and exercise prescription. $3^{\text {rd }}$ ed. Champaign (IL): Human Kinetics, 1998;121-44.

24. Raso V, Andrade EL, Matsudo SMM, Matsudo VKR. Adiposidade corporal em mulheres idosas de acordo com o nível de atividade física e o número de horas de TV. Rev Bras Med Esporte 1998;4:139-42.

25. Raso V, Andrade EL, Matsudo SMM, Matsudo VKR. Aerobic or muscle strength exercise improve the performance in activities diary living of elderly women. Med Sci Sports Exerc 1999;31:S1766.

26. Launer LJ, Harris T, Rumpel C, Madans J. Body mass index, weight change, and risk of mobility disability in middle-aged and older women. The epidemiologic follow-up study of NHANES I. JAMA 1994;271: 1093-8.

27. Visser M, Langlois J, Guralnik JM, Cauley JA, Kronmal RA, Robbins J, et al. High body fatness, but not low fat-free mass, predicts disability in older men and women: the Cardiovascular Health Study. Am J Clin Nutr 1998;68:584-90.

28. Visser M, Harris TB, Langlois J, Hannan MT, Roubenoff R, Felson DT, et al. Body fat and skeletal muscle mass in relation to physical disability in very old men and women of the Framingham Heart Study. J Gerontol A Biol Sci Med Sci 1998;53:M214-21.

29. Zamboni M, Turcato E, Santana H, Maggi S, Harris TB, Pietrobelli A, et al. The relationship between body composition and physical performance in older women. JAGS 1999;47:1403-8.

30. Zuliani G, Romagnoni F, Volpato S, Soattin L, Leoci V, Bollini MC, et al. Nutritional parameters, body composition, and progression of disability in older disabled residents living in nursing homes. J Gerontol A Biol Sci Med Sci 2001;56A:M212-6.

31. Friedman JM, Elasy T, Jensen GL. The relationship between body mass index and self-reported functional limitation among older adults: a gender difference. JAGS 2001;49:398-403.

32. Tully CL, Snowdon DA. Weight changes and physical function in older women: findings from the Nun Study. JAGS 1995;43:1394-7.

33. Landi F, Zuccala G, Gambassi G, Incalzi RA, Manigrasso L, Pagano F, et al. Body mass index and mortality among older people living in the community. J Am Geriatr Soc 1999;47:1072-6. 